\title{
Reasons for extended length of stay following chest tube removal in general thoracic surgical patients
}

\author{
Ammar Asban, Rongbing Xie, Peter Abraham, James K. Kirklin, James Donahue, Benjamin Wei \\ Department of Surgery, Division of Cardiothoracic Surgery, University of Alabama at Birmingham, Birmingham, Alabama, USA \\ Contributions: (I) Conception and design: B Wei; (II) Administrative support: R Xie, JK Kirklin; (III) Provision of study materials or patients: J \\ Donahue, B Wei; (IV) Collection and assembly of data: A Asban, P Abraham, R Xie; (V) Data analysis and interpretation: R Xie; (VI) Manuscript \\ writing: All authors; (VII) Final approval of manuscript: All authors. \\ Correspondence to: Benjamin Wei, MD. Associate Professor of Cardiothoracic Surgery, Program Director, Thoracic Surgery Residency, Zeigler \\ Research Building, 707, 19th St South, University of Alabama at Birmingham Medical Center, Birmingham, Alabama 35233, USA. \\ Email: bwei@uabmc.edu.
}

\begin{abstract}
Background: Many patients undergoing general thoracic surgery can be discharged on the same day as chest tube removal, but some are not, leading to increased resource utilization. This study assesses the frequency and duration of extended length of stay (ELOS) after tube removal and identifies risk factors for ELOS.

Methods: We retrospectively reviewed all adult patients undergoing general thoracic surgery at a tertiary referral medical center captured in the Society of Thoracic Surgeons General Thoracic Surgery Database and obtained detailed clinical data on chest tube management from August 2013 to April 2017. Pre-operative demographics, procedures, diagnoses, comorbidities, hospital service category, and lab values were examined to identify risk factors associated with ELOS after chest tube removal using multivariable generalized linear regression models.
\end{abstract}

Results: One thousand and four hundred seventy patients had $\geq 1$ chest tubes placed at the time of operation and discharged after chest tube removal: anatomic lung resection (34\%), wedge resection (29\%), decortication (16\%), and other (21\%). Fifty-one percent of these patients were male, $81 \%$ were white, and the mean age was 59 years (SD: 15 years). One-third of the patients had prior cardiothoracic operations. Twenty-three percent of these patients had ELOS, defined as discharge $\geq 1$ calendar day after chest tube removal with a median additional hospital stay of 3 days (interquartile range, 2-7 days). A multivariable regression model demonstrated that risk factors for ELOS included being admitted to an oncology or transplant service, undergoing decortication procedure, active smoking, and increased disability.

Conclusions: Patients with obesity, more severe disability, or actively smoking, undergoing, decortication, admitted to transplant and oncology services were more likely to experience ELOS. These factors should be considered when identifying appropriate patient groups for fast-track algorithms.

Keywords: Length of stay; chest tube; thoracic surgical surgery

Submitted Mar 08, 2020. Accepted for publication Aug 13, 2020.

doi: $10.21037 /$ jtd-20-1210

View this article at: http://dx.doi.org/10.21037/jtd-20-1210

\section{Introduction}

Similar to enhanced recovery pathways seen in other surgical specialties $(1,2)$. Fast-track pathways have been utilized in thoracic surgery to speed up recovery and reduce postoperative stay $(3-7)$. Chest tube management following thoracic surgical procedures plays a pivotal role in this process. Many patients are able to be discharged the same day as postoperative chest tube removal, while others require an extended length of stay (ELOS).

Many aspects of postoperative chest tube management 
have previously been studied, including the utility of intraoperative chest tube placement, the timing of chest tube removal, and the optimal output threshold for chest tube removal. Some studies show that foregoing postoperative chest tube drainage is not associated with major adverse events in specific patient populations (8). Although these results sound promising, they are not applicable to many patient populations and should not be taken to mean that chest tube drainage should be omitted entirely (9). The optimal timing of chest tube removal has long been debated, with many centers relying on volume output threshold as the benchmark by which to decide the appropriate time for chest tube removal $(4,10,11)$. Some centers have turned to digital devices for chest tube output volume calculations and air leak assessments, but the use of analog devices still remains popular in many institutions across the US (12-15). Appropriate thresholds for output volumes have also been studied, as some surgeons have argued for higher daily volume outputs $(450-500 \mathrm{cc})$ to be used as acceptable thresholds for chest tube removal (10). Together, these studies highlight some of the various details that should be considered when undergoing postoperative chest tube drainage.

However, despite the extensive research on postoperative chest tube management, there remains a paucity of literature surrounding the factors that influence discharge following chest tube removal. Some studies have mentioned sameday discharge following chest tube removal, but none have analyzed the barriers or facilitators to making this a reality $(16,17)$. This retrospective study at a tertiary referral center aims to provide further insight into those reasons for ELOS following chest tube removal. The goal of this study is to assess the frequency and duration of hospital stay after chest tube removal and identify specific risk factors associated with ELOS. We present the following article in accordance with the Strengthening the Reporting of Observational Studies in Epidemiology (STROBE) reporting checklist (available at http://dx.doi.org/10.21037/jtd-20-1210).

\section{Methods}

\section{Data source and patient selection}

This was a retrospective study using the Society of Thoracic Surgeons General Thoracic Surgery Database at the University of Alabama at Birmingham, a tertiary referral medical center, all adult patients who underwent a general thoracic surgery procedure between August 1,
2013 and April 1, 2017 were identified. Cases that involved intraoperative placement of a chest tube were deemed eligible and included. Esophagectomies were excluded. A total of 1,470 patients were included and followed up until their discharge. Informed consents are covered by general preoperative consent forms.

Demographic characteristics including age, race, gender, body mass index (BMI), smoking status (current, former, never), and activity status (ECOG score) were collected. Preoperative clinical characteristics such as comorbidities congestive heart failure (CHJTD-201210-R1F), coronary artery disease (CAD), peripheral vascular disease (PVD), transient ischemic attack (TIA), cerebrovascular accident (CVA), pulmonary hypertension, interstitial fibrosis, diabetes), preoperative diagnosis, steroid use, prior cardiothoracic surgery, current dialysis status, and history of chemotherapy or radiation were also collected. Postoperative variables recorded include procedure type, last Creatinine $(\mathrm{Cr}, \mathrm{mg} / \mathrm{dL})$, last Hemoglobin $(\mathrm{Hgb}$, $\mathrm{g} / \mathrm{dL}$ ), air leak $>5$ days, location of discharge (home, hospice, nursing home, other), postoperative pneumonia, readmission within 30 days, initial ventilator support requirement $>48 \mathrm{~h}$, postoperative urinary tract infection (UTI), postoperative catheterization or urinary retention, and discharge with urinary catheter. In this manuscript, our primary outcome, ELOS, was defined as staying in the hospital for 1 or more calendar day(s) after the last chest tube is removed. The length of time between chest tube removal and discharge was recorded after review of the electronic medical record and the associated ICD-9 and ICD-10 codes. Demographic and clinical data were then analyzed to identify any predictors for delayed discharge after chest tube removal. The study was conducted in accordance with the Declaration of Helsinki (as revised in 2013). IRB approval (protocol \#170516005) was obtained for the performance of this study.

\section{Statistical analysis}

Descriptive statistics were conducted, and continuous variables were expressed as median and interquartile range. Dichotomous variables/ordinal variables were expressed as number and proportion. Continuous variables were compared using $t$-test; dichotomous variables were compared using the chi-square test. To eliminate any rule of confounding factors or bias, univariate and then multivariable linear regression analysis were conducted to identify predictors associated with LOS after chest tube 
Table 1 Postoperative characteristics of patients experiencing ELOS after chest tube removal compared to patients with no ELOS

\begin{tabular}{|c|c|c|c|}
\hline Postoperative characteristics & $\begin{array}{l}\text { ELOS after CT removal } \\
(n=338)\end{array}$ & $\begin{array}{l}\text { No ELOS after CT removal } \\
(n=1,132)\end{array}$ & $P$ value \\
\hline Hospital days (median, IQR) & $7.0(4.0-14.0)$ & $2.0(1.0-4.0)$ & $<0.0001$ \\
\hline Duration of chest tube use (median, IQR) & $3.0(1.0-5.0)$ & $2.0(1.0-3.1)$ & $<0.0001$ \\
\hline Urinary retention req. Catheterization (n, \%) & $39(11.5)$ & $161(14.2)$ & 0.2046 \\
\hline Discharged with Foley catheter (n, \%) & $4(1.2)$ & $24(2.1)$ & 0.2682 \\
\hline Pneumonia (n, \%) & $28(8.3)$ & $18(1.6)$ & $<0.0001$ \\
\hline Discharge to extended care/transitional care unit (n, \%) & $50(14.8)$ & $10(0.9)$ & $<0.0001$ \\
\hline \multicolumn{4}{|l|}{ Discharge facility (n, \%) } \\
\hline Discharge to home & $271(80.2)$ & $1,115(98.6)$ & $<0.0001$ \\
\hline Discharge to nursing home & $6(1.8)$ & $0(0.0)$ & $<0.0001$ \\
\hline Discharge to hospice & $4(1.2)$ & $1(0.1)$ & 0.0024 \\
\hline Discharge to other facility & $4(1.2)$ & $3(0.3)$ & 0.0315 \\
\hline Readmission within 30 days after discharge $(\mathrm{n}, \%)$ & $48(14.3)$ & $78(6.9)$ & $<0.0001$ \\
\hline
\end{tabular}

This table demonstrates the result of univariate analysis that includes postoperative factors and the difference between those with and without ELOS. ELOS, extend length of stay; IQR, interquartile range.

removal. Since we are comparing two cohorts in order to determine how their characteristics differed, we did not conduct any subgroup analysis or sensitivity analysis. SAS 9.4 was used for statistical analysis.

\section{Results}

\section{Patient demographic and clinical characteristics}

Of the 1,470 patients who were eligible and included in the study, $51 \%$ of patients were male, $81 \%$ were white, and the mean age was 59 years (SD: 15 years). All patients were followed up to discharge. Of the total cohort, 338 (23.0\%) patients had ELOS following chest tube removal with a median additional stay after chest tube removal of 3 days (interquartile range, 2-7 days) (Table 1). Surgical procedures were distributed between anatomic lung resection (34\%), wedge resection (29\%), decortication (16\%), and $21 \%$ had another general thoracic surgical procedure (Table 2). By univariate analysis, ELOS patients were younger than nonELOS patients, with a mean age difference of 2.6 years between the two groups. Black patients were more likely to have ELOS compared to white patients [79/263 (30\%) vs. 255/1,187 (21\%); P=0.0027 (Table 3)].

Compared to patients without ELOS, those with ELOS were more likely to have heart failure, CAD, stroke, diabetes, renal failure, pulmonary hypertension, and severe chronic illness (Table 3). Patients with ELOS were more likely to have an urgent operation and have their service of origin be oncology, transplant, or pulmonary (Table 2). Among surgical procedures, decortication operations were particularly prone to ELOS (Table 2). Following surgery, ELOS patients were more likely to develop pneumonia, require longer mechanical ventilation, and develop urinary infections and sepsis (Table 1). In addition, ELOS patients were more likely to be discharged to a transitional care 
Table 2 Pre-operative and intraoperative characteristics of patients experiencing ELOS after chest tube removal compared to patients with no ELOS

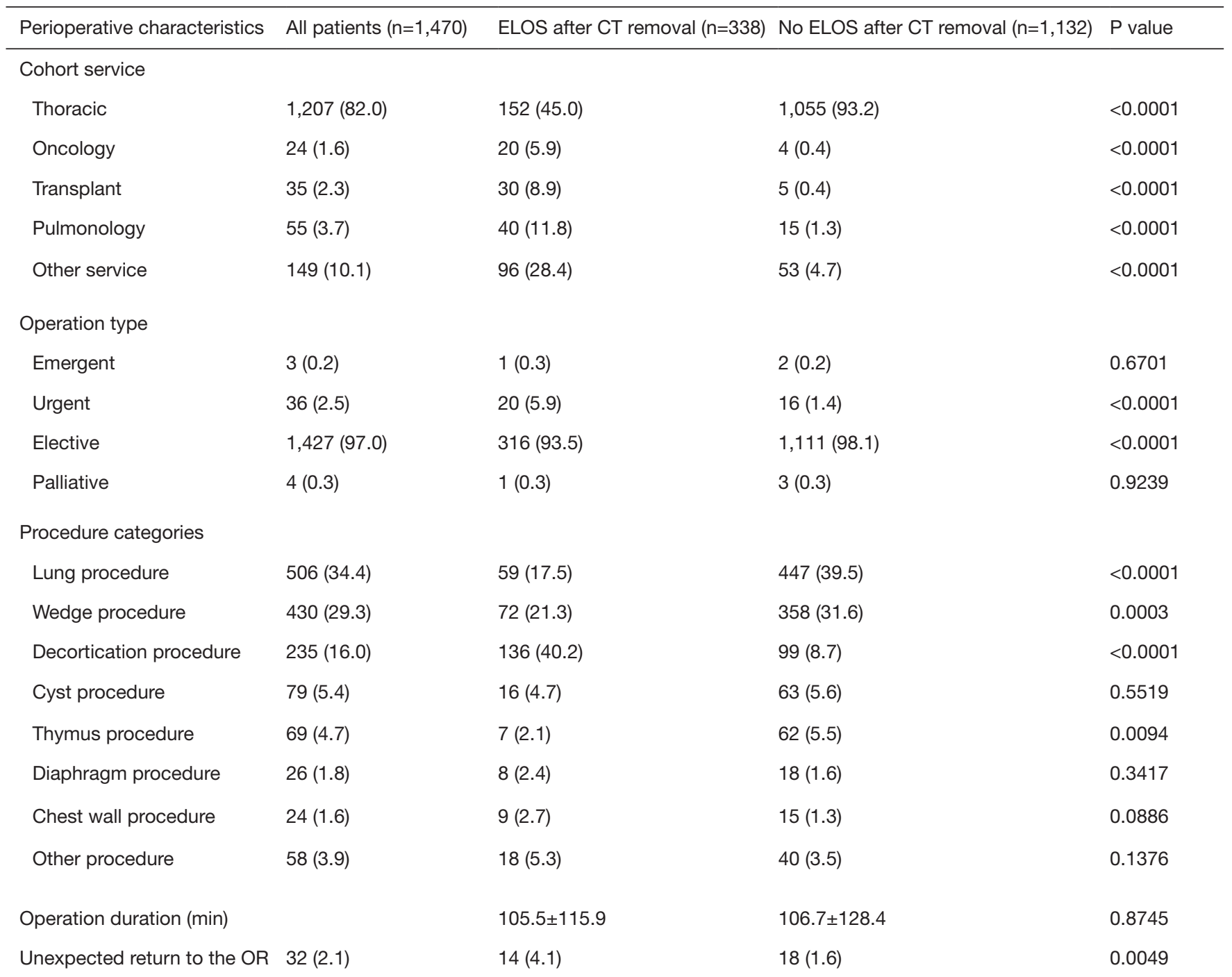

This table showed the result of univariate analysis that includes operative type, procedure type and service patients of those with and without ELOS. ELOS, extended length of stay.

facility or nursing home, and require readmission within 30 days (Table 1).

Of the 338 with ELOS, the majority of them (195 patients) stayed for one more day after chest tube removal $(57.6 \%)$. Figure 1 demonstrates the distribution of patient length of stay after chest tube removal.

\section{Multivariable analysis}

To examine the many factors associated with ELOS, a multivariable linear regression analysis examined predictors of duration of stay following chest tube removal was conducted. The three highest impact predictors of longer stay post chest tube removal include admission to a transplant service, admission to an oncology service, and greater generalized disability (Table 4). In terms of procedures, addition, patients undergoing decortication were at highest risk for ELOS after chest tube removal. As for comorbidities, obesity, anemia, and current smoking were the strongest predictors of ELOS after chest tube removal (Table 4). Use of robotic surgery, race, gender, and insurance status were not risk factors for ELOS. 
Table 3 Comparisons of ELOS after chest tube removal among thoracic surgery patients based on demographics and clinical characteristics

\begin{tabular}{|c|c|c|c|c|}
\hline Demographics & All patients $(n=1,470)$ & ELOS after CT removal $(n=338)$ & No ELOS after CT removal $(n=1,132)$ & $P$ value \\
\hline \multicolumn{5}{|l|}{ Sex } \\
\hline Male & $746(50.7)$ & $184(54.4)$ & $562(49.6)$ & 0.1221 \\
\hline \multicolumn{5}{|l|}{ Race } \\
\hline Black & $263(17.9)$ & $79(23.4)$ & $184(16.3)$ & 0.0027 \\
\hline BMI & $28.0 \pm 6.6$ & $28.6 \pm 7.1$ & $27.9 \pm 6.4$ & 0.0990 \\
\hline \multicolumn{5}{|l|}{ Smoking } \\
\hline Never & $544(37.0)$ & $132(39.1)$ & $412(36.4)$ & 0.3805 \\
\hline \multicolumn{5}{|l|}{ Comorbidities } \\
\hline Hypertension & $842(57.3)$ & $200(59.2)$ & $642(56.8)$ & 0.4323 \\
\hline Congestive heart failure & $84(5.7)$ & $42(12.4)$ & $42(3.7)$ & $<0.0001$ \\
\hline Coronary artery disease & $249(16.9)$ & $63(18.6)$ & $186(16.4)$ & 0.3456 \\
\hline Peripheral vascular disease & $83(5.6)$ & $21(6.2)$ & $62(5.5)$ & 0.6095 \\
\hline Transient ischemic attack (TIA) & $33(2.2)$ & $6(1.8)$ & $27(2.4)$ & 0.5052 \\
\hline Currently on dialysis & $31(2.1)$ & $18(5.3)$ & $13(1.1)$ & $<0.0001$ \\
\hline Diabetes & $280(19.0)$ & $85(25.1)$ & $195(17.2)$ & 0.0012 \\
\hline Steroid use & $150(10.2)$ & $50(14.8)$ & $100(8.8)$ & 0.0015 \\
\hline Prior cardiothoracic surgery & $526(35.8)$ & $128(37.9)$ & $398(35.2)$ & 0.3672 \\
\hline $\begin{array}{l}\text { Preoperative chemo - current } \\
\text { malignancy }\end{array}$ & $163(11.1)$ & $25(7.4)$ & $138(12.2)$ & 0.0136 \\
\hline $\begin{array}{l}\text { Preoperative thoracic radiation } \\
\text { therapy }\end{array}$ & $143(9.7)$ & $24(7.1)$ & $119(10.5)$ & 0.0626 \\
\hline \multicolumn{5}{|l|}{ ECOG status } \\
\hline Normal activity, no symptoms & $251(17.1)$ & $15(4.4)$ & $236(20.9)$ & $<0.0001$ \\
\hline
\end{tabular}

Table 3 (continued) 
Table 3 (continued)

\begin{tabular}{|c|c|c|c|c|}
\hline Demographics & All patients $(n=1,470)$ & ELOS after CT removal $(n=338)$ & No ELOS after CT removal $(n=1,132)$ & $P$ value \\
\hline $\begin{array}{l}\text { Symptoms, in bed } \leq 50 \% \text { of } \\
\text { time }\end{array}$ & $304(20.7)$ & $125(37.0)$ & $179(15.8)$ & $<0.0001$ \\
\hline $\begin{array}{l}\text { Symptoms, in bed }>50 \% \text { but } \\
\text { less than } 100 \% \text { of time }\end{array}$ & $98(6.7)$ & $52(15.4)$ & $46(4.1)$ & $<0.0001$ \\
\hline Bedridden & $25(1.7)$ & $22(6.5)$ & $3(0.3)$ & $<0.0001$ \\
\hline Moribund & $4(0.3)$ & $4(1.2)$ & $0(0.0)$ & 0.0002 \\
\hline Last creatinine level (mg/dL) & \pm 1.2 & \pm 1.6 & \pm 1.0 & $<0.0001$ \\
\hline Last hemoglobin level (g/dL) & $12.7 \pm 2.3$ & $11.2 \pm 2.7$ & $13.2 \pm 1.9$ & $<0.0001$ \\
\hline
\end{tabular}

This table demonstrates the result of univariate analysis that includes demographics and clinical characteristics of patients with ELOS and those without ELOS. ELOS, extend length of stay.

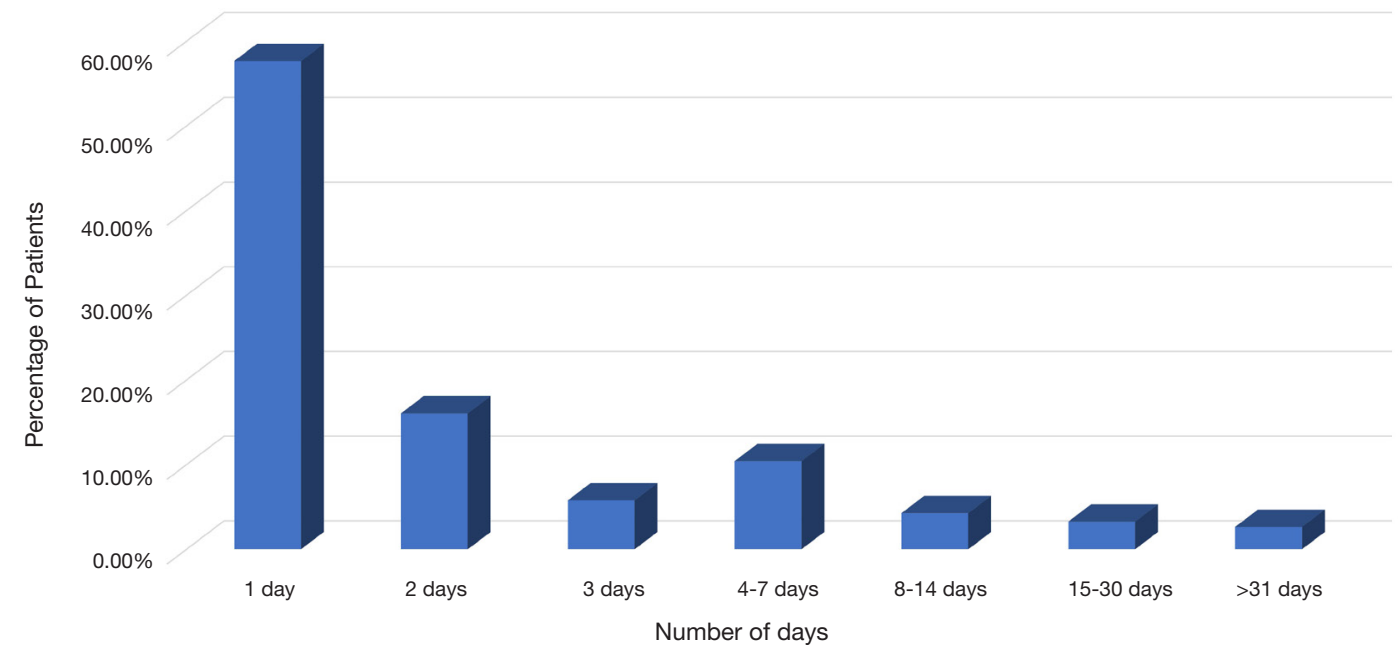

Figure 1 Distribution of extended stay in days among patients with ELOS ( $\mathrm{n}=338)$. ELOS, extended length of stay.

\section{Discussion}

The results of this study show that the service into which a patient is admitted may serve as a predictor for ELOS. In our institution, patients admitted to transplant, pulmonary, and oncology services were more likely to experience
ELOS after chest tube removal than patients admitted to general thoracic surgery services. In addition, certain patient characteristics such as obesity, severe disability, or active smoking were associated with increased length of stay following chest tube removal and should be considered when determining postoperative pathways to discharge. Our 
Table 4 Predictors (by multivariable linear regression) of ELOS after chest tube removal among fast-track thoracic surgery patients

\begin{tabular}{lll}
\hline Risk factor & Coefficient $^{\mathrm{a}}$ & $\mathrm{P}$ value \\
\hline Service transplantation & 7.88 & $<0.0001$ \\
Service oncology & 2.10 & 0.015 \\
Greater disability (Zubrod $\geq 2)$ & 1.42 & $<0.0001$ \\
Decortication procedure & 1.31 & 0.0004 \\
Current smoker & 1.05 & 0.0001 \\
Lower hemoglobin (per $1 \mathrm{~g} / \mathrm{dL})$ & 0.41 & $<0.0001$ \\
Higher BMl (per $\left.5 \mathrm{~kg} / \mathrm{m}^{2}\right)$ & 0.31 & 0.001 \\
\hline
\end{tabular}

This table illustrates risk factors that are associated with extended length of stay based on multivariable linear regression analysis. ${ }^{a}$, Number of additional days of extended length of stay associated with presence of risk factor. ELOS, extend length of stay.

finding that decortication procedures were associated with ELOS indicates that the type of thoracic surgery procedure should also be considered when constructing fast-track pathways.

Our study adds to current literature surrounding postoperative chest tube management by exploring what factors affect the timeline of a patient's discharge following chest tube removal. We were unsurprised to find that nonthoracic surgical services had higher rates of ELOS, as chest tube management is protocolized and familiar to our thoracic surgery team. Discharge planning often centers on the date of anticipated removal of the chest tube, a parameter that is best understood by the surgical team. Similarly, the comorbidities that correlated with higher ELOS—current smoking, obesity, severe disability—were also unsurprising as these conditions have been shown to lead to longer lengths of stay and increased complications for patients undergoing thoracic procedures (18-22).

Finding differences in ELOS among procedure type was interesting as it suggests there may be utility in creating fasttrack pathways that are procedure-specific and not broadly applied to all general thoracic surgical procedures. The increased length of stay for decortication procedures may be explained by the pathology of disease, as decortication procedures may be undertaken after long bouts of illness or chronic disease as opposed to wedge resections or other anatomic lung resections which may be done on an elective basis. Some patients require prolonged administration of intravenous antibiotics following decortication for empyema, which requires additional planning and could partially explain ELOS in this population.

The main limitation of this study is that it was performed at a single institution and thus may be subject to institutionspecific biases. For example, the difference seen in ELOS between the thoracic service and other services may not be seen at other institutions depending on their chest tube protocol and education practices. However, our cohort is a representative sample and therefore, we believe that the findings of this study would be applicable to other institutions.

\section{Conclusions}

We found that there are certain risk factors that are associated with the ELOS following chest tube removal. These include obesity, severe disability, active smoking, admission to non-thoracic surgical services, and undergoing decortication procedures. These factors should be considered when establishing fast-track algorithms, as pathways may need to be adjusted to accommodate for patients possessing these risk factors. Utilizing expedited pathways to minimize ELOS following chest tube removal could help optimize resource utilization in the care of general thoracic surgical patients.

\section{Acknowledgments}

This paper was presented as a poster presentation at the 32nd Annual Meeting of the General Thoracic Surgical Club, March 7-10, 2019, Naples, FL.

Funding: None.

\section{Footnote}

Reporting Checklist: The authors have completed the STROBE reporting checklist. Available at http://dx.doi. org/10.21037/jtd-20-1210

Data Sharing Statement: Available at http://dx.doi. org/10.21037/jtd-20-1210

Conflicts of Interest: All authors have completed the ICMJE uniform disclosure form (available at http://dx.doi. org/10.21037/jtd-20-1210). JKK reports other from Society of Thoracic Surgeons, outside the submitted work; BW serves as an unpaid editorial board member of fournal of Thoracic Disease from Oct 2018 to Sep 2020. The other 
authors have no conflicts of interest to declare.

Ethical Statement: The authors are accountable for all aspects of the work in ensuring that questions related to the accuracy or integrity of any part of the work are appropriately investigated and resolved. The study was conducted in accordance with the Declaration of Helsinki (as revised in 2013). IRB approval (protocol \#170516005) was obtained for the performance of this study. Informed consents are covered by general preoperative consent forms.

Open Access Statement: This is an Open Access article distributed in accordance with the Creative Commons Attribution-NonCommercial-NoDerivs 4.0 International License (CC BY-NC-ND 4.0), which permits the noncommercial replication and distribution of the article with the strict proviso that no changes or edits are made and the original work is properly cited (including links to both the formal publication through the relevant DOI and the license). See: https://creativecommons.org/licenses/by-nc-nd/4.0/.

\section{References}

1. Rawlinson A, Kang P, Evans J, et al. A systematic review of enhanced recovery protocols in colorectal surgery. Ann R Coll Surg Engl 2011;93:583-8.

2. Spanjersberg WR, Reurings J, Keus F, et al. Fast track surgery versus conventional recovery strategies for colorectal surgery. Cochrane Database Syst Rev 2011:CD007635.

3. Brunelli A. Enhanced recovery pathways in thoracic surgery: Time for a version 2.0. J Thorac Cardiovasc Surg 2018;155:2758-9.

4. Cerfolio RJ, Bryant AS. Does minimally invasive thoracic surgery warrant fast tracking of thoracic surgical patients? Thorac Surg Clin 2008;18:301-4.

5. D'Andrilli A, Rendina EA. Enhanced recovery after surgery (ERAS) and fast-track in video-assisted thoracic surgery (VATS) lobectomy: preoperative optimisation and care-plans. J Vis Surg 2018;4:4.

6. Jones NL, Edmonds L, Ghosh S, et al. A review of enhanced recovery for thoracic anaesthesia and surgery. Anaesthesia 2013;68:179-89.

7. Loop T. Fast track in thoracic surgery and anaesthesia: update of concepts. Curr Opin Anaesthesiol 2016;29:20-5.

8. Ueda K, Hayashi M, Tanaka T, et al. Omitting chest tube drainage after thoracoscopic major lung resection. Eur J
Cardiothorac Surg 2013;44:225-9; discussion 9.

9. Dumans-Nizard V, Guezennec J, Parquin F, et al. Feasibility and results of a fast-track protocol in thoracic surgery. Minerva Anestesiol 2016;82:15-21.

10. Cerfolio RJ, Bryant AS. Results of a prospective algorithm to remove chest tubes after pulmonary resection with high output. J Thorac Cardiovasc Surg 2008;135:269-73.

11. Cerfolio RJ, Bryant AS, Skylizard L, et al. Optimal technique for the removal of chest tubes after pulmonary resection. J Thorac Cardiovasc Surg 2013;145:1535-9.

12. Cerfolio RJ, Bryant AS. The benefits of continuous and digital air leak assessment after elective pulmonary resection: a prospective study. Ann Thorac Surg 2008;86:396-401.

13. Reeb J, Falcoz PE, Olland A, et al. Are daily routine chest radiographs necessary after pulmonary surgery in adult patients? Interact Cardiovasc Thorac Surg 2013;17:995-8.

14. Tanaka T, Ueda K, Murakami J, et al. Use of Stitching and Bioabsorbable Mesh and Glue to Combat Prolonged Air Leaks. Ann Thorac Surg 2018;106:e215-e8.

15. Varela G, Jiménez MF, Novoa NM, et al. Postoperative chest tube management: measuring air leak using an electronic device decreases variability in the clinical practice. Eur J Cardiothorac Surg 2009;35:28-31.

16. Bjerregaard LS, Jensen K, Petersen RH, et al. Early chest tube removal after video-assisted thoracic surgery lobectomy with serous fluid production up to $500 \mathrm{ml} /$ day. Eur J Cardiothorac Surg 2014;45:241-6.

17. Tovar EA, Roethe RA, Weissig MD, et al. One-day admission for lung lobectomy: an incidental result of a clinical pathway. Ann Thorac Surg 1998;65:803-6.

18. Groth SS, Whitson BA, Kuskowski MA, et al. Impact of preoperative smoking status on postoperative complication rates and pulmonary function test results 1-year following pulmonary resection for non-small cell lung cancer. Lung Cancer 2009;64:352-7.

19. Johnston G, Goss JR, Malmgren JA, et al. Health status and social risk correlates of extended length of stay following coronary artery bypass surgery. Ann Thorac Surg 2004;77:557-62.

20. McDevitt J, Kelly M, Comber H, et al. A population-based study of hospital length of stay and emergency readmission following surgery for non-small-cell lung cancer. Eur J Cardiothorac Surg 2013;44:e253-9.

21. Musallam KM, Rosendaal FR, Zaatari G, et al. Smoking and the risk of mortality and vascular and respiratory events in patients undergoing major surgery. JAMA Surg 
2013;148:755-62.

22. Musallam KM, Tamim HM, Richards T, et al.

Preoperative anaemia and postoperative outcomes in

Cite this article as: Asban A, Xie R, Abraham P, Kirklin JK, Donahue J, Wei B. Reasons for extended length of stay following chest tube removal in general thoracic surgical patients. J Thorac Dis 2020;12(10):5700-5708. doi: 10.21037/jtd20-1210 non-cardiac surgery: a retrospective cohort study. Lancet 2011;378:1396-407. 